\title{
Pseudomonas in the sinks in an intensive care unit: relation to patients
}

\author{
MARK H LEVIN, BRUCE OLSON, CATHERINE NATHAN, SHERWIN A KABINS, \\ ROBERT A WEINSTEIN
}

From the Infection Control Program and Division of Infectious Diseases, Michael Reese Hospital and
Medical Center and the Pritzker School of Medicine, University of Chicago, Chicago, Illinois 60616

SUMMARY Sink drains in a medical-surgical intensive care unit (ICU) were cultured during six consecutive weeks as part of a seven month prospective study of acquisition of Pseudomonas aeruginosa by ICU patients. Isolates were typed serologically and by aminoglycoside and chlorhexidine susceptibility patterns. All 11 sinks contained multiple strains of $P$ aeruginosa; some strains persisted for weeks while others were isolated once. Of the sink isolates $56 \%$ had high level resistance to gentamicin and tobramycin whereas none of the strains found in patients. In sink isolates chlorhexidine resistance correlated with aminoglycoside resistance and with the presence of a chlorhexidine dispenser at a sink. The sequence of recovery of phenotypically similar isolates suggested that sinks were the source of at most two acquisitions of $P$ aeruginosa by patients during the six weeks. Our study confirms that sinks may be reservoirs for large numbers of highly resistant $P$ aeruginosa but are rarely the source of organisms colonising patients in our ICU.

The use of barrier precautions to prevent the spread of organisms resistant to aminoglycosides among patients in hospital has controlled resistant enterobacteriaceae but not Pseudomonas aeruginosa in our hospital. ${ }^{2}$ The reasons for this discrepancy are not clear. To understand better the acquisition of $P$ aeruginosa, we prospectively studied patients admitted to a medical-surgical intensive care unit (ICU) (Olson B, unpublished observations). As part of that study we extensively cultured potential environmental sources for $P$ aeruginosa. In particular we included a detailed study of sinks because they often harbour antibiotic resistant bacteria ${ }^{3-6}$ yet no definitive study has prospectively established the relative contribution of sink contamination to nosocomial pseudomonas. We report here the prevalence in sinks of $P$ aeruginosa resistant to aminoglycosides and chlorhexidine, the relation of chlorhexidine use to sink contamination, and the extent to which patient colonisation could be traced to sink isolates.

\section{Material and methods}

CULTURES

The medical-surgical ICU at Michael Reese HospiAccepted for publication 3 January 1984 tal is a 12 bed unit with 11 sinks. Five of the sinks are in the patient care area and six are in adjacent medication, lounge, and laboratory rooms. All 11 sinks are often used by ICU personnel caring for patients. Chlorhexidine dispensers are used at six of the sinks.

Sink drains (traps) were sampled weekly during the last six weeks of a seven month prospective study of ICU patients. Drain specimens were obtained by passing a Culturette swab (Marion Scientific Corporation, Rockford, Illinois) $10 \mathrm{~cm}$ into the trap and swabbing the walls until the tip was soiled. The material was plated on MacConkey and cetrimide-containing agar (Pseudosel Agar, BBL, Cockeysville, Maryland). Five colonies were randomly selected from the Pseudosel agar and subcultured on MacConkey and tryptic soy agar. Lactose negative, oxidase positive isolates were serotyped (Difco, Detroit, Michigan). Strains that reacted with a specific antisera were considered to be $P$ aeruginosa. Strains that autoagglutinated or did not agglutinate were tested for ability to metabolise acetamide and gluconate. ' Isolates which metabolised both were considered to be $P$ aeruginosa. Isolates were stored on tryptic soy agar at $4^{\circ} \mathrm{C}$.

Several other environmental sources were also sampled during the six weeks: these included patient 
care equipment, such as respiratory treatment devices, suction tubing and bottles, and measuring cylinders; medical equipment, such as stethoscope heads, ophthalmoscope handles, and manometers; frequently handled environmental surfaces, such as intravenous poles, telephone handles, bedside computer keyboards, bed guard rails, and soap dishes; faucet aerators; and feedings.

All patients admitted to the ICU for more than $24 \mathrm{~h}$ had rectal and throat cultures taken on admission, once weekly, and on discharge. The results of all clinical cultures were also reviewed. All isolates of $P$ aeruginosa were serotyped.

\section{SUSCEPTIBILITY TESTING}

After four to nine weeks of storage, one isolate of each serotype (from each sink) was selected for aminoglycoside susceptibility testing by an agar dilution method. ${ }^{8}$ Thirty two of the 42 strains were viable for testing. A strain was considered susceptible to gentamicin or tobramycin if the minimum inhibitory concentration (MIC) was $\leqslant 5.0 \mu \mathrm{g} / \mathrm{ml}$, low level resistant if the MIC was 10 or $20 \mu \mathrm{g} / \mathrm{ml}$, and high level resistant if the MIC was $\geqslant 40 \mu \mathrm{g} / \mathrm{ml}$. Susceptibility to amikacin was defined as an MIC $\leqslant 10 \mu \mathrm{g} / \mathrm{ml}$ and low level resistance as an MIC of $20 \mu \mathrm{g} / \mathrm{ml}$.

Chlorhexidine diacetate (Sigma, St Louis, Missouri) susceptibility testing was performed by an agar dilution method ${ }^{9}$ on strains for which aminoglycoside susceptibility data were available. Strains with an MIC of $\geqslant 100 \mu \mathrm{g} / \mathrm{ml}$ of chlorhexidine were considered resistant; ${ }^{10}$ almost all had MICs $\geqslant 400$ $\mu \mathrm{g} / \mathrm{ml}$. Twenty six of 32 strains were still viable after 10-15 weeks of storage and were tested. Two high level gentamicin and tobramycin resistant, one low level gentamicin resistant, and three aminoglycoside susceptible strains were not viable at the time of chlorhexidine testing.

\section{CRITERIA FOR DETERMINING THE RELATION}

BETWEEN SINK AND PATIENT ISOLATES

It was considered that a patient may have acquired a $P$ aeruginosa strain from a sink if the following criteria were met: the patient's and the sink isolate had the same serotype and aminoglycoside susceptibility pattern; the strain was found in a sink before it was cultured from the patient (because of the compact size of the unit and the frequent use of all sinks, the exact proximity of the sink to the patient was not a factor); and the strain had not been isolated from any other patient or other environmental source just before or during the period of acquisition.

\section{Results}

\section{SINK DRAIN CULTURES}

Two hundred and five strains of $P$ aeruginosa were isolated from 11 sinks during the six week period. There were 12 different serotypes; up to four were found in any one drain and two to six were isolated from each sink over the six weeks. Some serotypes were repeatedly cultured from a sink; others appeared only once. If repeat isolates of the same serotype from individual sinks were excluded, 42 strains were isolated (Table 1). Cultures of faucet aerators (present on only three sinks) did not grow pseudomonas.

\section{SUSCEPTIBILITY TESTING}

Eighteen $(56 \%)$ of the 32 viable strains had high level resistance to gentamicin and tobramycin. Low level resistance to amikacin was found in five of these 18. Low level gentamicin resistance was found in four other strains, one of which also had low level

Table 1 Serotypes of $P$ aeruginosa cultured from sinks and patients in the ICU during six week study

\begin{tabular}{|c|c|c|c|}
\hline Serotype & No of sinks & No of patients infected while in ICU & $\begin{array}{l}\text { No of patients already colonised on } \\
\text { admission }\end{array}$ \\
\hline 1 & - & - & 1 \\
\hline $1,9,10$ & $1(2)$ & 2 & 1 \\
\hline $2,5,16$ & $2(5)$ & - & 2 \\
\hline 3 & $1(2)$ & - & - \\
\hline 4 & $4(10)$ & - & 1 \\
\hline 6 & $6(14)$ & - & 7 \\
\hline 7,8 & $2(5)$ & - & - \\
\hline 10 & $3(7)$ & 2 & 1 \\
\hline 11 & 7 (17) & 1 & 1 \\
\hline 13,14 & $5(12)$ & - & 1 \\
\hline 14 & $1(2)$ & - & - \\
\hline Cross react & $3(7)$ & - & 1 \\
\hline Non typeable & $7(17)$ & - & 1 \\
\hline Total & $42 *(100)$ & $5 \dagger$ & $17 \ddagger$ \\
\hline
\end{tabular}

${ }^{*}$ Eighteen $(56 \%)$ of 32 strains tested had high level resistance to gentamicin and tobramycin.

†None of these strains had high level resistance to gentamicin or tobramycin. Two of the five (a serotype 10 and 11$)$ were possibly acquired from sinks based on similar susceptibility patterns and the temporal sequences of isolation (see Methods and Results).

$\ddagger$ None of these strains had high level resistance to gentamicin or tobramycin. 
Table 2 Correlation of aminoglycoside (Am) and chlorhexidine (Chl) susceptibility of P aeruginosa from ICU sinks and presence of chlorhexidine dispensers

\begin{tabular}{|c|c|c|c|c|}
\hline & \multicolumn{4}{|c|}{ No of sink isolates with the following susceptibilities } \\
\hline & $\begin{array}{l}\text { Chl resistant } \\
(+ \text { Am resistant })\end{array}$ & $\begin{array}{l}\text { Chl sensitive } \\
(+ \text { Am sensitive })\end{array}$ & $\begin{array}{l}\text { Am resistant } \\
(+ \text { Chl sensitive })\end{array}$ & $\begin{array}{l}\text { Am sensitive } \\
(+C h l \text { resistant })\end{array}$ \\
\hline $\begin{array}{l}\text { From sinks with a Chl } \\
\text { dispenser } \\
\text { From sinks without a Chl } \\
\text { dispenser } \\
\text { Total }\end{array}$ & $\begin{array}{cc}19 \dagger & (15) \\
4 \dagger & (4) \\
& (19) \S\end{array}$ & $\begin{aligned} 0 \dagger & (0) \\
3+ & (3) \\
& (3) 8\end{aligned}$ & $\begin{aligned} 16 \neq & (0) \\
6 \ddagger & (0) \\
& (0) 8\end{aligned}$ & $\begin{array}{ll}5 \ddagger & (4) \\
5 \ddagger & (0) \\
& (4) 8\end{array}$ \\
\hline
\end{tabular}

*Am resistant includes high and low level resistance to gentamicin and tobramycin as defined in Materials and methods section.

†Correlation of Chl resistant with presence of Chl dispenser, $p=0.01$ (Fisher's exact test).

$\ddagger$ Correlation of Am resistant with presence of Chl dispenser, $p=0 \cdot 20$.

$\S$ Correlation of Chl resistant with Am resistant $(2 \times 2$ contingency table: $19,0,4,3), p=0.01$.

resistance to amikacin.

Twenty three $(88 \%)$ of 26 strains were resistant to chlorhexidine (Table 2). Chlorhexidine resistance correlated significantly with aminoglycoside resistance $(p=0.01)$ and with the presence of a chlorhexidine dispenser at a sink $(p=0 \cdot 01)$. There was an association between aminoglycoside resistance in sink isolates and the presence of a chlorhexidine dispenser, but this was not significant.

Based on the patterns of aminoglycoside and chlorhexidine susceptibilities, the 12 serotypes of $P$ aeruginosa in our sinks could be subdivided into at least 18 different strains.

\section{STRAINS OF P AERUGINOSA ISOLATED FROM} SINKS AND PATIENTS

During the six weeks, 60 patients were admitted to the ICU for more than $24 \mathrm{~h}$ and five acquired $P$ aeruginosa (Table 1). None of the five acquisitions was due to cross infection (as determined by review of both surveillance and clinical culture results). Two of the patient strains, however, were phenotypically similar to strains previously isolated from sinks (a serotype 10 and an 11, susceptible to gentamicin, tobramycin, and amikacin) and thus may have been acquired from the sinks. Other environmental sources seemed unlikely since cultures of only four of 129 patient care objects or environmental surfaces that we sampled grew pseudomonas-an Ambu bag, a feeding tube connector, and two suction catheters, all of which were from the immediate environment of patients already colonised.

\section{Discussion}

We found widespread contamination of our ICU sink drains with $P$ aeruginosa. Based on serotyping and aminoglycoside and chlorhexidine susceptibilities there were at least 18 strains, some persisting in sinks for weeks while others were recovered only once. Although the occurrence of $P$ aeruginosa in sink traps is well documented, ${ }^{3-6}$ we found many more strains than have been reported when only a single phenotypic marker is used. ${ }^{6}$

The prevalence of aminoglycoside resistance in our sink isolates (Table 1,2 ) confirms a previous observation that environmental gram negative bacilli may be very resistant. ${ }^{5}$ Our isolates also showed a high frequency of resistance to chlorhexidine, which occurred with aminoglycoside resistance to a significant level, suggesting that an antiseptic dispensed at sinks might select out multiply resistant environmental strains. Although further observations in control wards would be needed to test this hypothesis, others have noted a similar phenomenon in patients-association of antibiotic and antiseptic resistance in gram negative bacilli isolated from the urine of paraplegic men after chronic meatal exposure to chlorhexidine.9"1

The interest in contaminated drains stems from a concern that bacteria in sinks might be a source of infections in patients. Bacteria may be splattered from contaminated sinks onto the hands of personnel during washing and thereby be spread to patients. ${ }^{3}$ Teres et $\mathbf{l}^{6}$ found that $6 \%$ of patients in their respiratory care unit acquired $P$ aeruginosa with pyocin types similar to isolates from the unit's sinks. That study, however, did not include antibiotic or antiseptic susceptibility testing as markers to distinguish strains further. In fact, in that unit control of sink flora by periodic sterilisation was not effective in decreasing patient infection, suggesting that sinks were not of epidemiological importance. ${ }^{12}$ Perryman $e t a^{5}$ similarly believed that sink drains could not be incriminated epidemiologically in their hospital; however, they looked only at clinical (and not surveillance) culture data in a retrospective fashion and did not focus on any particular pathogen as a marker of transmission.

In our seven month prospective survey of ICU 
patients, $36(13 \%)$ of 270 patients developed rectal or throat carriage of $\boldsymbol{P}$ aeruginosa. Only two of these 36 strains, and none of the five strains acquired by patients during the six week sink survey (Table 1), were resistant to high levels of gentamicin and tobramycin. This is in contrast to the high level resistance often found in sink isolates and suggests little clinical importance of the sink contamination. At most two of the five strains acquired by ICU patients during the six week sink study matched the serotype and susceptibility patterns of strains that had been found in the sinks. Our methods of distinguishing strains of $\boldsymbol{P}$ aeruginosa (serotyping and susceptibility patterns) are relatively crude, however, and use of additional markers might have shown even greater disparity between sink and patient isolates.

In conclusion, ICU sink drains harboured a frequently changing range of $P$ aeruginosa. Over half the strains had high level resistance to gentamicin and tobramycin. Chlorhexidine use at sinks correlated with chlorhexidine resistance in drain isolates and possibly with aminoglycoside resistanceobservations that warrant further investigation. Serotyping, susceptibility data, and the temporal pattern of isolates suggested that patients at worst only occasionally acquire strains from sinks. In fact, many more patients arrived at our ICU already colonised with pseudomonas (Table 1), reinforcing the concept that sink related strains are a relatively minor component of the endemic ICU ecology.

\section{References}

' Gaynes RP, Weinstein RA, Smith J, Carman M, Kabins SA.
Control of aminoglycoside resistance by barrier precautions. Infect Control 1983;4:221-4.

${ }^{2}$ Weinstein RA, Nathan C, Gruensfelder R, Kabins SA. Endemic aminoglycoside resistance in gram-negative bacilli: epidemiology and mechanisms. J Infect Dis 1980;141:338-45.

${ }^{3}$ Brown DG, Baublis J. Reservoirs of Pseudomonas in an intensive care unit for newborn infants: mechanisms of control. $J$ Pediatr 1977;90:453-7.

4 Moody MR, Young VM, Kenton DM, Vermeulen GD. Pseudomonas aeruginosa in a Center for Cancer Research. I. distribution of intraspecies types from human and environmental sources. J Infect Dis 1972;125:95-101.

${ }^{5}$ Perryman FA, Flournoy DJ. Prevalence of gentamicin- and amikacin-resistant bacteria in sink drains. J Clin Microbiol 1980;12:79-83.

- Teres O, Schweers P, Bushnell LS, Hedley-Whyte J, Feingold DS. Sources of $P$ aeruginosa infection in a respiratory-surgical intensive therapy unit. Lancet $1970 ; \mathrm{i}: 415-7$.

' Pickett MJ, Pederson MM. Nonfermentative bacilli associated with man: II. detection and identification. Am J Clin Pathol 1970;54:164-77.

${ }^{8}$ Washington JA II. The agar-dilution technique. In: Balows A ed. Current techniques for antibiotic susceptibility testing, Springfield, Illinois: Charles C Thomas, 1974:54-62.

9 Stickler DJ, Thomas B, Jagdish JC. Antiseptic and antibiotic resistance in gram-negative bacteria causing urinary tract infection in spinal cord injur:d patients. Paraplegia 1981;19:50-8.

${ }^{10}$ Nakahara $\mathrm{H}$, Kozukue $\mathrm{H}$. Isolation of chlorhexidine-resistant Pseudomonas aeruginosa from clinical lesions. J Clin Microbiol 1982;15: 166-8.

"Stickler DJ, Thomas B. Antiseptic and antibiotic resistance in gram-negative bacteria causing urinary tract infection. J Clin Pathol 1980;33:288-96.

${ }^{12}$ Feeley TW, DuMoulin GC, Hedley-Whyte J, Bushnell LS, Gilbert JP, Feingold DS. Aerosol polymixin and pneumonia in seriously ill patients. $N$ Engl J Med 1975;193:471-5.

Requests for reprints to: Dr Robert A Weinstein, Department of Medicine, Michael Reese Hospital and Medical Center, Lake Shore Drive at 31st Street, Chicago, Illinois 60616, USA. 